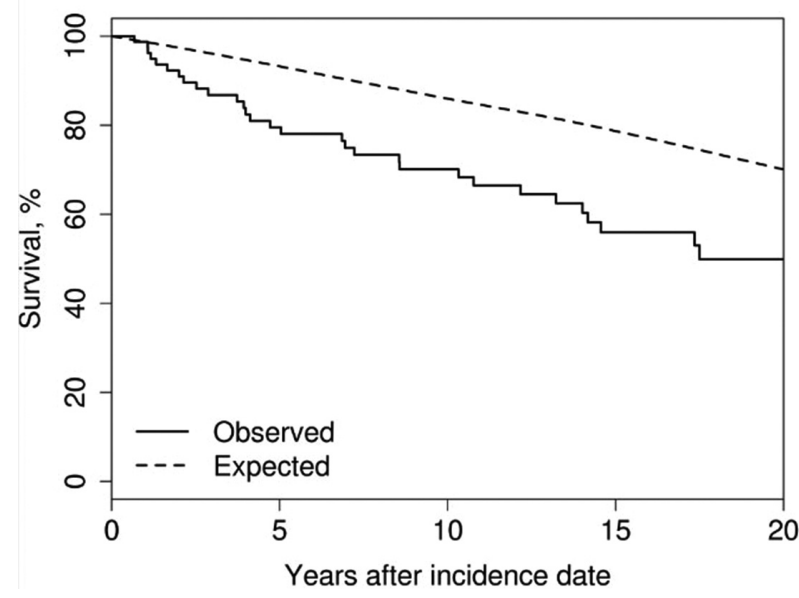

Conclusions: The average incidence of SSc in this population-based US cohort was 2.7 per 100000 population with no change in incidence over the 36 year period of study. The new 2013 classification criteria perform significantly better than the 1980 criteria, but failed to classify $19 \%$ of patients in this cohort. Overall survival of patients with SSc is worse than the general population with no evidence of improved survival over time, indicating an unmet need for early diagnosis and more aggressive management.

Disclosure of Interest: None declared

DOI: 10.1136/annrheumdis-2018-eular.3853

\section{SAT0477 INTENSIFIED B-CELL DEPLETION THERAPY IN PROGRESSIVE SYSTEMIC SCLEROSISPATIENTS: 24 MONTHS FOLLOW-UP}

D. Rossi, I. Cecchi, S. Sciascia, M. Radin, E. Rubini, D. Roccatello. Center of Immunopathology and Rare Diseases, University of Torino, Torino, Italy

Background: Systemic sclerosis (SSc) is a connective tissue autoimmune disease with systemic involvement and a serious medical condition with a high rate of mortality, especially due to interstitial lung disease (ILD). The exact pathophysiology is still unclear, but B cells seem to play a crucial role in the initiation and the progression of the disorder. ${ }^{1}$ Therefore, the use of Rituximab (RTX) might have a rational in the treatment of SSc.

Objectives: We aimed to investigate the outcomes of SSc patients treated with RTX after a follow-up of 24 months.

Methods: We retrospectively collected data from SSc patients ${ }^{2}$ resistant or intolerant to previous therapies, treated with intensified B-depletion therapy, between 2013 and 2016. Therapeutic protocol comprehends: RTX $375 \mathrm{mg} / \mathrm{sm}$ on days 1 , $8,15,22$, and two more doses after one and two months, associated with two intravenous administrations of $10 \mathrm{mg} / \mathrm{kg}$ of cyclophosphamide and three methylprednisolone pulses $(15 \mathrm{mg} / \mathrm{kg})$ followed by oral prednisone $(0.8 \mathrm{mg} / \mathrm{kg} / \mathrm{day}$, rapidly tapered to $5 \mathrm{mg} /$ day by the end of the 3rd month after RTX).

Results: he study included 20 SSc patients (18 females and 2 males; mean age $66.7 \pm 11.0$ years). Patients presented with severe multiorgan involvement: ILD $(19 / 20,95 \%)$, pulmonary hypertension $(12 / 20,60 \%)$, and skin thickening $(17 / 20$, $85 \%$ ). After a follow-up of 24 months, we observed a decrease in the levels of NTproBNP (mean baseline: $385.4 \pm 517$, mean at 24 months: $283 \pm 648$, $p<0.05$ ), and in the Modified Rodnan Skin Score (mRSS) (mean mRSS baseline: $14.4 \pm 10.5$, mean after 24 months of follow-up: $12.9 \pm 10, p<0.05)$. Four out $19(21 \%)$ patients experienced a significant improvement of ILD, as assessed by high-resolution computed tomography, while in 12/19 (63\%) patients the intensified B-cell depletion therapy was associated with a stabilisation of the imaging features with no sign of progression. Three out of $19(16 \%)$ patients showed a deterioration of the ILD.

Patients showed no significant decrease in forced vital capacity (FVC) (mean baseline FVC: $93.6 \pm 19.3$, mean after 24 months of follow-up: 92.2 \pm 23.3 ), no significant decrease in forced expiratory volume in one second (FEV1) (mean base-

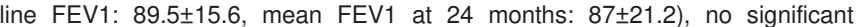
decrease in diffusing capacity (DLCO) (mean baseline DLCO values: 58.8 \pm 8.6 , mean at 24 months: $60.3 \pm 14$ ), no significant change in the ejection fraction (EF) (mean baseline EF values: $62.8 \pm 6.4$, mean EF values at 24 months: $58.6 \pm 7.1$ ) and in pulmonary artery pressure (PAP) (mean baseline PAP: $30.2 \pm 10.5$, mean at 24 months: $31.1 \pm 11.05)$.

Conclusions: Despite recent advances in the treatment of SSc, ILD heavily affects prognosis and life expectancy of these patients. Our data suggest that the intensified B-depletion therapy protocol might represent a promising tool for the management of SSc in terms of controlling the progression of the disease, especially when considering pulmonary and skin manifestations. Further prospective studies are needed in order to confirm our results.

\section{REFERENCES:}

[1] Forestier A, Guerrier T, Jouvray M, Giovannelli J, Lefèvre G, Sobanski V, et al. Altered B lymphocyte homeostasis and functions in systemic sclerosis. Autoimmun Rev 2018.

[2] Pope JE, Johnson SR. New Classification Criteria for Systemic Sclerosis (Scleroderma). Rheum Dis Clin North Am 2015;41:383-98.

Disclosure of Interest: None declared

DOI: 10.1136/annrheumdis-2018-eular.6241

\section{SAT0478 DISEASE-SPECIFIC AUTOANTIBODIES ASSOCIATE WITH REMARKABLY DIFFERENT RISK OF DEVELOPMENT OF SIGNIFICANT LUNG FIBROSIS IN SYSTEMIC SCLEROSIS}

A. Sari, S.I. Nihtyanova, A. Gill, V.H. Ong, C.P. Denton. Rheumatology, Royal Free Hospital, University College London, London, UK

Background: Pulmonary fibrosis (PF) is an important complication of systemic sclerosis (SSc), being a leading cause of disease related death. Some studies suggest that the timing of PF development differs between patients with different autoantibodies

Objectives: We set out to assess a large single-centre SSc cohort, focusing on the timing of clinically significant PF (csPF), and to compare this within subgroups with different disease-specific autoantibodies, in particular anti-centromere antibody (ACA), anti-topoisomerase I antibody (ATA) and anti-RNA polymerase antibody (ARA).

Methods: Patients with confirmed SSc and information on autoantibodies were included. PF was confirmed on high-resolution $\mathrm{CT}$ and defined as clinically significant based on at least one of the following: FVC $<70 \%$; a drop in $\mathrm{FVC}>15 \%$; DLCO $<70 \%$ with no pulmonary hypertension $(\mathrm{PH})$ present; or a drop in DLCO $>15 \%$ with no $\mathrm{PH}$. Only subjects who had first available lung function test result within the first 3 years from onset were included. 1-Kaplan-Meier (1 KM) estimation was used to calculate cumulative incidence of csPF. To assess the timing of highest rates of csPF development, hazard rates were calculated within intervals of 12 months over the follow-up.

Results: A total of 450 subjects, 75 (16.7\%) male, mean age of onset 47.4 years, were included in the study. Of those $225(50 \%)$ had diffuse cutaneous SSc, 105 (23.3\%) carried ACA, $113(25.1 \%)$ ATA and 72 (16\%) ARA. Mean follow-up was 12 years, interquartile range 8-16 years. Over the entire follow-up period, 196 $(43.6 \%)$ of the subjects developed csPF.

Using $1 \mathrm{KM}$ estimation, for the whole cohort, over the first 20 years of disease, approximately half of the patients developed csPF. Three quarters of the patients who developed csPF had reached this endpoint by 5 years (38.2\%) with much lower incidence thereafter (at year 10, 15 and $20 \%-43 \%, 47 \%$ and $49.6 \%$, respectively)

Analysis within subgroups showed that, ACA was associated with a very low risk of csPF development (cumulative incidence of $5.9 \%, 8.1 \%, 9.8 \%$ at 5,10 and 15 years from SSc onset). On the other hand, ATA + patients had a remarkably high risk of csPF development, which ultimately occurred in the majority of cases, with cumulative incidence of $77.6 \%$ at 5 years, $82.7 \%$ at 10 years and $87.1 \%$ at 15 years. Rates of csPF development among ARA+patients were higher than those in ACA+, but still much lower than ATA+, and even after 20 years of follow-up, the cumulative incidence of csPF among them was less than a half of that among ATA + patients $(23.7 \%, 33 \%$ and $41 \%$ at years 5,10 and 15 , figure 1$)$.

The hazard of cSPF among ACA + patients was highest in the second year from SSc onset $(3 \%)$ and in the subsequent years varied between $0 \%$ and $1.8 \%$. On the other hand, among ATA + patients hazard of csPF was $28.3 \%$ in year $1,44.9 \%$ in year 2, peaked at $52.5 \%$ in year 3 and went down sharply thereafter. Although hazard was much lower among ARA+patients, this still peaked at year $3(2.8 \%$, $6.1 \%$ and $12.1 \%$ at year 1,2 and 3 respectively) and declined after. 


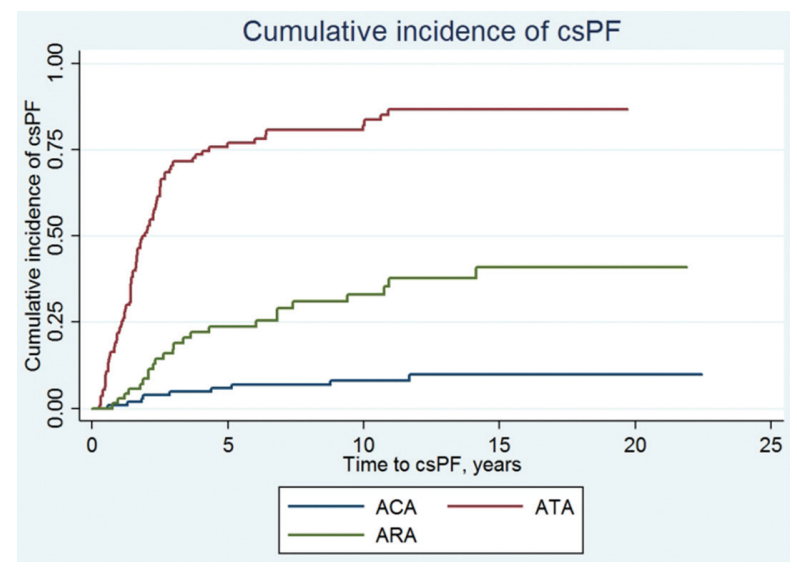

Conclusions: Our analysis demonstrates that csPF is a complication that tends to develop early in the disease course. Although the overall risk of csPF differs by antibodies, it is highest at around 3 years from disease onset and goes down thereafter. This can be used to inform organ disease monitoring and clinical trials recruitment.

Disclosure of Interest: None declared

DOI: 10.1136/annrheumdis-2018-eular.7089

\section{SAT0479 LASER SPECKLE CONTRAST ANALYSIS: A PILOT STUDY AND SYSTEMATIC LITERATURE REVIEW OF RELIABILITY OF THE QUANTITATIVE ASSESSMENT OF PERIPHERAL BLOOD PERFUSION IN SYSTEMIC SCLEROSIS}

A. Vanhaecke ${ }^{1,2}$, M. Cutolo $^{3}$, B. Ruaro ${ }^{3}$, L. Deroo ${ }^{1}$, C. Ickinger $^{4}$, K. Melsens ${ }^{1,2}$ Y. Piette ${ }^{2}$, F. De Keyser ${ }^{1,2}$, V. Smith ${ }^{1,2}$ on behalf of the EULAR study group on microcirculation in Rheumatic diseases. ${ }^{1}$ Department of Internal Medicine, Ghent University; ${ }^{2}$ Department of Rheumatology, Ghent University Hospital, Ghent, Belgium; ${ }^{3}$ Research Laboratory And Academic Division Of Clinical Rheumatology, Department Of Internal Medicine, Irccs San Martino Aou, University Of Genoa, Genoa, Italy; ${ }^{4}$ Division of Rheumatology, Faculty of Health Sciences, University of the Witwatersrand, Johannesburg, South Africa

Background: Microvasculopathy is an important feature of systemic sclerosis (SSc), making its assessment a key issue in SSc clinical research. Nailfold videocapillaroscopy (NVC) is a valuable tool to detect and classify microvascular structural alterations. In contrast, laser speckle contrast analysis (LASCA), a noninvasive microvascular imaging tool, has been proposed as an objective technique to dynamically evaluate the peripheral blood perfusion (PBP).

Objectives: The specific objectives were 1) to perform a pilot study to investigate as first both the intra- and inter-rater reliability of LASCA in an unselected SSc cohort and descriptively in healthy subjects (HS) and 2) to identify the available literature on the reliability of LASCA in SSc by a systematic literature review.

Methods: First, a pilot study was performed to assess the reliability of LASCA to measure the PBP at the level of the fingers in an unselected cohort SSc patients and descriptively in HS. Intra-rater reliability was assessed by having a first anchor rater performing the measurements at 2 time-points (within $15 \mathrm{~min}$ ) and inter-rater reliability by subsequently having the first anchor rater and a team of $3 \mathrm{~s}$ raters performing the measurements in $15 \mathrm{SSc}$ and $30 \mathrm{HS}$ (see figure 1). As external validation, the measurements were repeated with a second anchor rater in a distinct cohort of 15 SSc patients. Reliability was described by calculating the intraclass correlation coefficient (ICC).

The systematic search was performed to identify relevant full-text articles in PubMed, EMBASE and Web of Science. All retrieved articles were screened on title, abstract and full-text level, reference lists were additionally searched.

Results: Thirty SSc patients ( 5 men, 25 women; mean age $52 \pm 17$ y; 1 LSSc, 25LcSSc, 4DcSSc; 14 vasodilatory therapy) and $30 \mathrm{HS}$ (8 men, 22 women; mean age $33 \pm 11 \mathrm{y}$ ) underwent LASCA measurements. ICC for intra-rater reliability of the first anchor was $0.95(95 \% \mathrm{Cl} 0.86-0.98)$ in SSc and $0.93(95 \% \mathrm{Cl} 0.83-0.97)$ in HS, the ICC for inter-rater reliability was $0.97(95 \% \mathrm{Cl} 0.90-0.99)$ in SSc and 0.93 in HS. Intra- and inter-rater reliability of anchor 2 was $0.78(95 \% \mathrm{Cl} 0.46-$ $0.92)$ and $0.87(95 \% \mathrm{Cl} 0.67-0.96)$ respectively.

The systematic search identified 64 unique articles, of which 12 were eligible for full-text review. Two additional references were identified through a reference search of retrieved articles. Only 1 of the 14 selected references that met the inclusion criteria documented reliability as outcome and was included in the final analysis. This pilot study by Lambrecht et al measured the PBP at the level of the fingertips and reported ICC values varying from $0.82-0.91$ for the dorsal and $0.74-0.86$ for the volar fingertips.

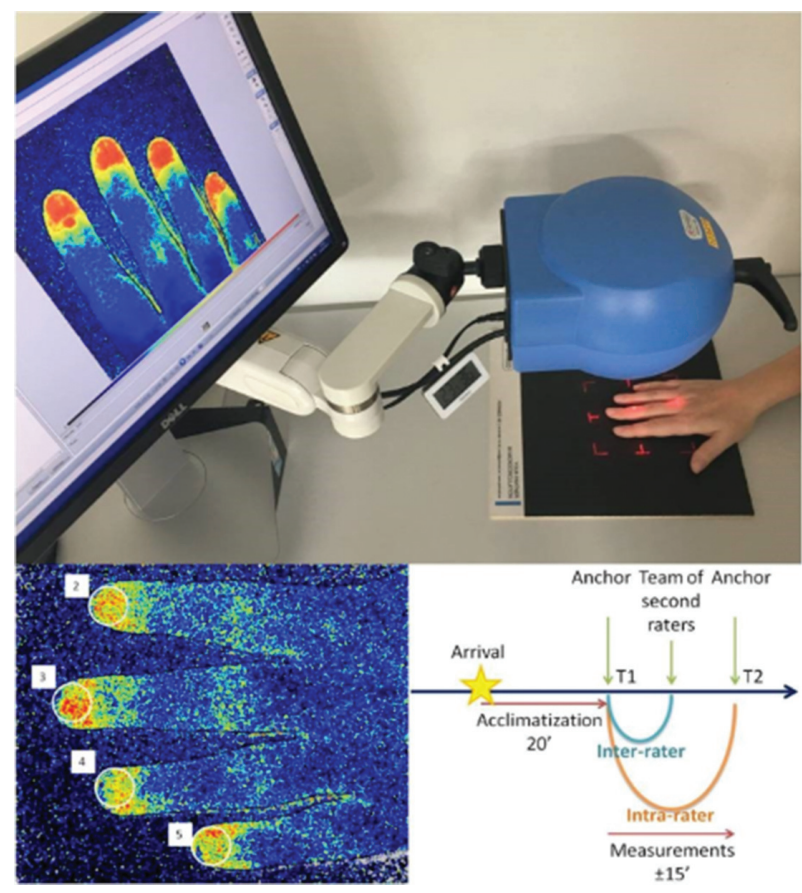

Conclusions: The body of knowledge regarding intra- and inter-rater reliability of LASCA in SSc is very limited. Only one manuscript reporting very good inter-rater reliability of PBP measurements of the distal fingertips by LASCA could be withheld. These results could be confirmed by our pilot study. In addition, we demonstrated excellent intra-rater reliability of LASCA measurements for the evaluation of the PBP of the hands in SSc patients and HS.

Disclosure of Interest: None declared

DOI: 10.1136/annrheumdis-2018-eular.3953

\section{SAT0480 EVALUATION OF STANDARDISED TEACHING OF MODIFIED RODNAN SKIN SCORE ASSESSMENT IN SYSTEMIC SCLEROSIS}

A.H. Low ${ }^{1,2}$, S.-A. $\mathrm{Ng}^{1,2}$, V. Berrocal ${ }^{3}$, B. Brennan ${ }^{3}$, G. Chan 4 , S.-C. Ng ${ }^{1,2}$, D. Khanna ${ }^{5} .{ }^{1}$ Rheumatology and Immunology, Singapore General Hospital; ${ }^{2}$ DukeNational University of Singapore, Singapore, Singapore; ${ }^{3}$ Biostatistics, University of Michigan, Michigan, USA; ${ }^{4}$ Rheumatology, Allergy and Immunology, Tan Tock Seng Hospital, Singapore, Singapore; ${ }^{5}$ Scleroderma Program, University of Michigan, Michigan, USA

Background: The modified Rodnan skin score (mRSS) is a standard outcome measure for skin involvement in systemic sclerosis (SSc) clinical trials. Training assessors reduces variability in mRSS measurement.

Objectives: Our objective is to report the inter- and intra-observer variability of mRSS scoring using newly developed standardised training guidelines by the Scleroderma Clinical Trials Consortium (SCTC).

Methods: Two SSc experts (DK/AL), 2 facilitators, 52 rheumatology trainees and 8 SSc patients fulfilling the 2013 American College of Rheumatology criteria participated in a SSc skin scoring workshop. Eight SSc patients were examined by 2 SSc experts and facilitators together and consensus scores reached. All trainees attended a talk on mRSS skin scoring by an SSc expert (DK), followed by a video and live demonstration by an expert examining a patient exhibiting different aspects of skin scoring. Each trainee subsequently performed mRSS scoring on 4 SSc patients independently. This concluded the teaching session for mRSS scoring. The mRSS scoring for each trainee was compared to the consensus expert mRSS, and a score of $\leq 5$ in 3 out of 4 patients is considered acceptable interobserver variability, as determined by SCTC guidelines.

Two days after training, 12 trainees, 2 facilitators and 2 experts re-assessed independently the mRSS of $2 \mathrm{SSc}$ patients whom they had examined previously. The repeat day $2 \mathrm{mRSS}$ score for each trainee was compared to the baseline mRSS score, and a score of $\leq 3$ is considered acceptable intra-observer variability.

We computed the inter- and intra-observer variability using a linear mixed model with an intercept term and random effects for patient, rater and patient by rater with the following values representing the degrees of agreement: $<0-$ poor; $0-0.20$ -slight; 0.21 - 0.40 -fair; $0.41-0.60$-moderate; $0.61-0.80$-substantial; and 0.81 1.00 -almost perfect agreement.

Results: For the first group of assessors involving 52 trainees, $65.4 \%$ of them achieved acceptable inter-observer variability, with inter-observer variability of 